\title{
Characterizing Severely Plastically Deformed Materials Using Transmission Kikuchi Diffraction and Energy Dispersive X-ray Spectroscopy in the Scanning Electron Microscope
}

\author{
Patrick Trimby
}

\section{Australian Centre for Microscopy \& Microanalysis, The University of Sydney, Sydney, Australia}

There has been considerable interest in severe plastic deformation (SPD) in recent years, primarily due to the desirable physical properties associated with grain size refinement. Advanced processing techniques, such as equal channel angular pressing (ECAP) and high pressure torsion (HPT), can produce materials with grain sizes in the nanostructured regime (i.e. with mean grain sizes below $100 \mathrm{~nm})$. Characterizing such microstructures is difficult. The combination of ultrafine grain sizes and high dislocation densities makes conventional electron backscatter diffraction (EBSD) particularly challenging. Some recent studies have focused on improving the spatial resolution of EBSD by utilizing lower energy electron beams (e.g. under $10 \mathrm{kV}$ ), but this can cause additional problems with drift and stability [1]. New automated diffraction techniques in the transmission electron microscope (TEM), notably using precession diffraction methods [2], have generated significant interest. Although the resolution of such approaches has been demonstrated to be in the range of 1-2nm using a field emission gun (FEG) TEM, there remain a number of issues relating to the reliability of diffraction pattern indexing, with additional problems encountered when the grain size is smaller than the sample thickness. This has limited analyses to relatively small areas or to samples with larger grain sizes.

Recent developments $[3,4]$ have shown how a standard EBSD system in a field emission gun scanning electron microscope (SEM) can be used for electron diffraction in transmission mode on electron transparent TEM samples. Initially this technique was referred to as "transmission EBSD" [3], but SEMbased transmission Kikuchi diffraction (TKD) is a more accurate term [4]. All of the early applications of TKD indicate a true spatial resolution in the order of $5-10 \mathrm{~nm}$, almost an order of magnitude better than conventional EBSD and close to that achieved using TEM-based techniques. This improved spatial resolution enables effective orientation mapping of even the most highly deformed materials, such as those developed using SPD techniques. Numerous examples will be shown in this paper, all collected using a standard commercial EBSD-EDS system fitted to a FEG-SEM, using a 30kV beam energy and typically $1-10 \mathrm{nA}$ beam current. The samples were mostly standard TEM foils, between 50 and $150 \mathrm{~nm}$ in thickness, mounted in a microclamp fitted to a pretilted sample holder (figure 1). The set up for TKD also enables imaging using standard forescatter detectors mounted on the bottom of the EBSD detector, and is ideal for simultaneous energy dispersive X-ray spectroscopy (EDS) analyses.

The forescatter detectors can be used to generate either phase/thickness contrast or orientation contrast images of the TEM samples. An example oriented dark field (ODF) image of an HPT-deformed NiCo alloy is shown in figure 1. The sample thickness and geometry enables simultaneous EDS analyses with a lateral spatial resolution in the order of $20-30 \mathrm{~nm}$. This is sufficient to map elemental segregation in SPD-processed intermetallic materials, and to correlate chemical variations with grain boundaries on the nanoscale. An example elemental map ( $\mathrm{Ni} \mathrm{Ka}$ ) from the same deformed NiCo alloy is shown in figure 2, and can be compared with the corresponding TKD pattern quality map and orientation map. This dataset is typical of the results from automated TKD / EDS mapping, and a range of SPD-processed samples have been successfully characterized using this approach, including duplex stainless steels, Al alloys, 
$\mathrm{Ni}$, Ti and various intermetallics. Measurement step sizes were typically in the $2 \mathrm{~nm}$ to $10 \mathrm{~nm}$ range, with collection speeds of $50-100 \mathrm{~Hz}$.

This study shows the potential for TKD in the SEM, with associated EDS analyses and forescatter detector ODF imaging, to characterize highly deformed and nanostructured materials using a standard FEG-SEM equipped with a commercial EDS-EBSD system. This accessibility and flexibility, coupled with the sub-10nm resolution, makes it a viable alternative to automated TEM diffraction techniques [5].

\section{References:}

[1] D. Chen et al., Ultramicroscopy 111 (2011), p. 1488-1494.

[2] P. Moeck et al., Cryst. Res. Technol. 46, (2011), p. 589-606

[3] R.R. Keller and R.H. Geiss, J. Microscopy, 245 (2012), p. 245-251.

[4] P.W. Trimby, Ultramicroscopy, 120 (2012), p. 16-24.

[5] The author acknowledges help from colleagues at Sydney University, including Adam Sikorski, Zibin Chen, Yang Cao, Gareth Davies, Xiaozhou Liao, Peter Liddicoat, Jihong Qiu and Jingli Sun.
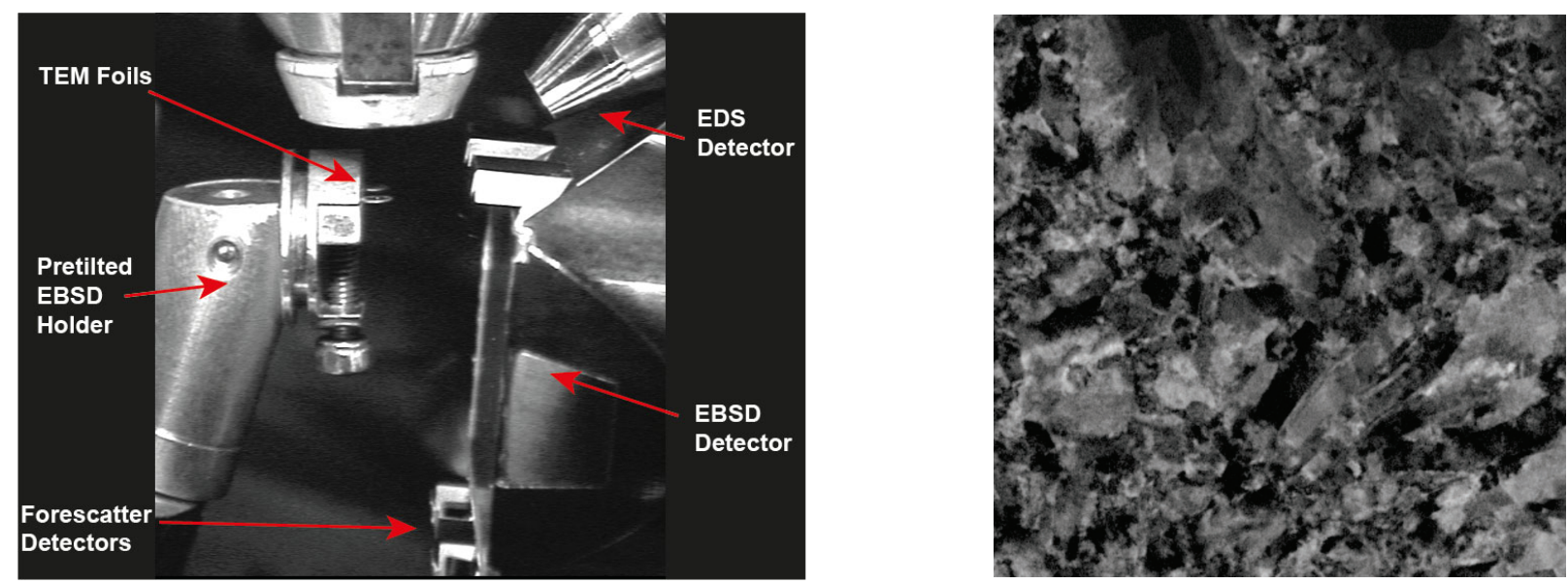

Figure 1. Left: SEM chamberscope image showing the standard configuration for TKD/EDS analyses. Right: Forescatter ODF image of an HPT deformed NiCo alloy. Field of view is $2.5 \mu \mathrm{m}$ across.
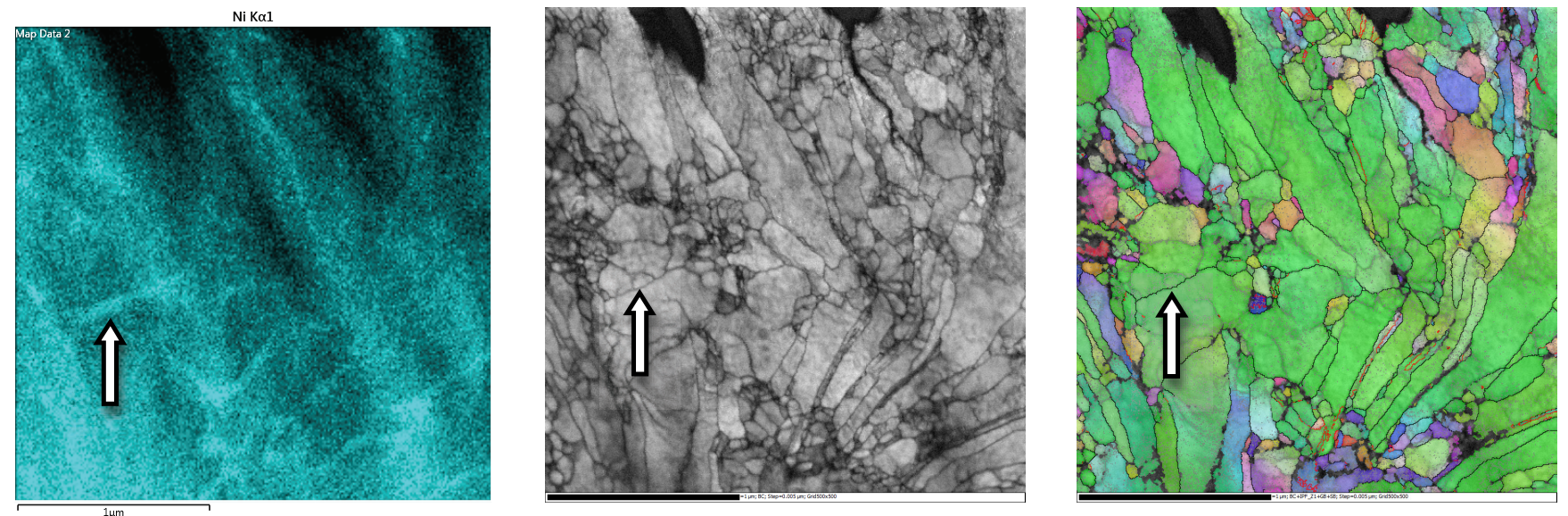

Figure 2. Left: Element map of the NiCo sample shown in figure 1, showing the Ni distribution. Centre: TKD pattern quality map of the same area (500x500 grid, 5nm step). Right: Inverse pole figure orientation map. Arrows indicate the same grain boundary in all images. All scale bars represent $1 \mu \mathrm{m}$. 